\title{
Radio/Antenna Mounting System for Wireless Networking under Row-Crop Agriculture Conditions
}

\section{Daniel K. Fisher}

U.S. Department of Agriculture, Agricultural Research Service, Crop Production Systems Research Unit, 141 Experiment Station Rd., Stoneville 38776, USA; E-Mail: daniel.fisher@ars.usda.gov; Tel.: +1-662-686-5342; Fax: +1-662-686-5422

Academic Editor: Dharma P. Agrawal

Received: 19 March 2015 / Accepted: 10 June 2015 / Published: 1 July 2015

\begin{abstract}
Interest in and deployment of wireless monitoring systems is increasing in many diverse environments, including row-crop agricultural fields. While many studies have been undertaken to evaluate various aspects of wireless monitoring and networking, such as electronic hardware components, data-collection procedures, power management, and communication protocols, little information related to physical deployment issues has been reported. To achieve acceptable wireless transmission capability, the radio/antenna must be positioned properly relative to the ground surface or crop canopy to minimize degradation of the radio signal, usually requiring the mounting of the radio/antenna above the canopy. This results in the presence of obstacles to normal agricultural equipment traffic and production operations and potential damage to the wireless monitoring system. A simple and rugged radio/antenna mounting system was designed which could be subjected to encounters with agricultural equipment without suffering physical damage. The mounting system was deployed and tested, and operated successfully following repeated encounters with various agricultural machines and implements. The radio/antenna mount is simple and inexpensive to fabricate using locally available components.
\end{abstract}

Keywords: wireless network; radio; antenna; row-crop agriculture

\section{Introduction}

Interest in and deployment of automated sensing and monitoring systems and wireless networks has been increasing rapidly in recent years. Many studies have been undertaken to develop and implement 
automated monitoring systems and wireless networks in agricultural and other diverse environments, as outlined in reviews such as those of Wang et al. [1] and Abbasi et al. [2]. The focus of much of the work reported has been on the development of the instrumentation and network itself, including electronic hardware components, sensing elements, data-collection procedures, power management, and communication protocols. Performance of the networks has often been reported in terms of data-transfer rates, number of successful transmissions, battery life, and transmission distance.

A critical component in a wireless network is the radio, which includes the electronic hardware (radio and antenna) as well as the physical placement of the radio and antenna. To achieve acceptable radio-transmission performance, factors which could improve or degrade transmissions, including topography, obstacles, and height above a reflecting surface, such as the ground surface or crop canopy, need to be taken into consideration. Radio waves travel between transmitting and receiving radios in a three-dimensional space called the Fresnel zone, which is defined by the distance between the radios and the frequency of the radio waves. Obstacles or reflecting surfaces in this zone can degrade radio signals and affect radio transmissions, so the radio antennas must be positioned at an appropriate height such that the majority of the Fresnel zone is above these influences.

Little information related to physical field deployment issues is usually provided in reports on wireless networking, however. The location of the networking equipment must be planned so that it is not an obstacle or in danger of suffering damage during normal production operations. In some installations, such as in a greenhouse, orchard, or vineyard, deployment issues may be minimal due to the presence of fixed structures, such as walls, fences, trees, or trellises, and the equipment can be installed on or near these. Workers operating in these environments are aware of these fixed objects, and the network devices are unlikely to be damaged.

In other circumstances, such as in cultivated fields under row-crop conditions, there are few, if any, structures in the field due to the need for agricultural equipment to access the fields and perform various operations throughout the growing season. The installation of monitoring and wireless data-transmission equipment in the field, therefore, introduces obstacles to normal field operations and movement of agricultural vehicular equipment. Encounters between field equipment, such as tractors, implements, and chemical applicators, and wireless networking components could result in damage to the electronic instrumentation. To avoid damage, workers could remove or lay down the instrumentation prior to passage of agricultural equipment, repositioning it upon completion of the field operation, but this is labor- and time-consuming, and could result in loss of data while waiting for safe re-entry conditions into the field. Alternative efforts by equipment operators to avoid damaging the instrumentation, such as driving around the installation or raising equipment above the obstacle, could result in under-treated regions and non-representative growing conditions in the monitored area.

The objective of this work was to develop and test a radio/antenna mount for wireless monitoring systems which could be deployed in row-crop agricultural fields and be subjected to encounters with agricultural field equipment without being an obstacle to field operations or suffering physical damage.

\section{Design of the Mounting System}

The design of the radio/antenna mounting system was dictated by the needs of achieving satisfactory data-transmission performance while minimizing impact on field operations. Mounting requirements 
included: (1) placement of the radio above the canopy for improved line-of-sight and data-transmission range, and (2) simple, rugged, and flexible construction so as to withstand encounters with equipment during routine field operations, such as cultivation, and fertilizer and other chemical applications.

A diagram of the radio/antenna mount which was developed is shown in Figure 1. The mount was designed to support a tall mast, with a small radio or radio antenna, attached to the top of the mast. Under normal conditions, the mast would remain in a vertical orientation to provide sufficient height above the ground surface or crop canopy. If agricultural equipment, such as a tractor pulling an implement, were operating in the field and encountered the radio mast, the mast would pivot and pass under the implement. The tension spring would be extended, and when the implement had passed, tension in the spring would return the mast to the vertical orientation.
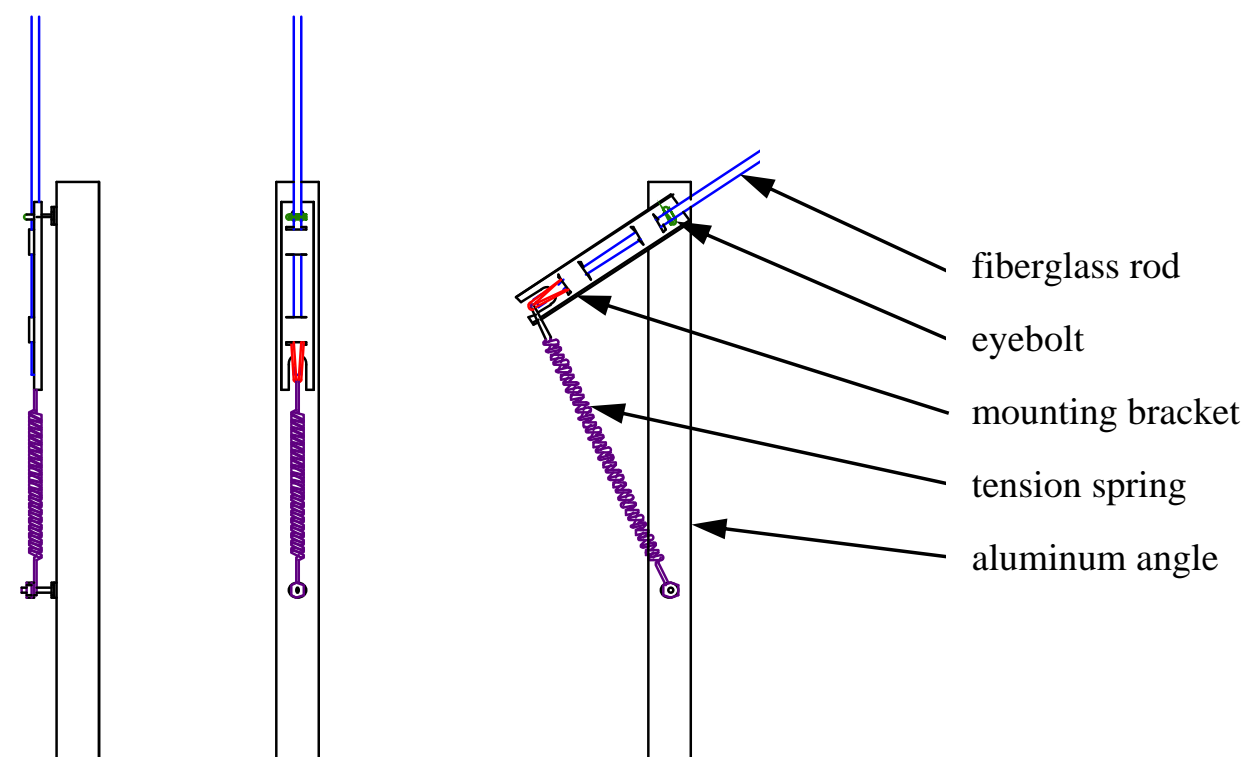

Figure 1. Side (Left) and front (Middle) views of the radio/antenna mounting system in the normal, unflexed orientation, and flexed, under strain (Right).

The mounting system consisted of a length of aluminum angle stock, flexible fiberglass pole, eyebolt, tension spring, and mounting hardware. The mount was fabricated by first cutting the $32 \mathrm{~mm} \times 32 \mathrm{~mm}$ aluminum angle stock to a length of $60 \mathrm{~cm}$. A hole was drilled through one side of the angle stock approximately $3 \mathrm{~cm}$ from one end, and a second hole was drilled $29 \mathrm{~cm}$ from the first. The fiberglass pole, available commercially as a flag for use on a bicycle to improve visibility, consisted of a fiberglass rod $2 \mathrm{~m}$ in length and an orange flag. A mounting bracket attached to the pole, used to fasten the flag to a bicycle, was removed from the rod. An eye bolt was inserted into a hole in the bracket, and a mating nut was threaded onto the bolt. The eyebolt was inserted into the upper hole in the aluminum angle and secured with a second mating nut. The fiberglass rod was inserted through the eye of the eyebolt and reinstalled in the mounting bracket. The tension spring, approximately $10 \mathrm{~cm}$ long with a tension rating of $1.3 \mathrm{~kg}$ (force), was attached by first inserting a bolt through the loop on one end of the spring and into the bottom hole in the aluminum angle, where it was secured with a mating nut. The spring was then attached to the bottom of the pole's mounting bracket via a loop of steel wire.

The fabrication details provided above were based on the particular components available locally to the author. Part numbers and manufacturers of the components are not provided because they are not 
critical in the fabrication of the radio/antenna mount, and other similar components could be substituted to construct an equally functional mounting system. A wooden stake or flat steel bar, for example, could be used in place of the aluminum angle stock. If a flag pole with a mounting bracket were not available, two small hose clamps, one attached to the pole above the eyebolt and the other attached to the bottom end of the pole, could be used to secure the pole and connect the tension spring. Keeping the design simple and using locally available components results in a device which could be replicated or modified easily and inexpensively (total cost of the mounting system described in this report was approximately US\$10).

\section{Deployment and Testing}

Radio/antenna mounts were fabricated and deployed at the USDA Agricultural Research Service's Crop Production Systems Research Unit farm at Stoneville, Mississippi USA during the 2014 growing season. The mounting systems were used to test a wireless monitoring instrument, a modification of a datalogging device designed to automate soil-moisture sensor measurements [3]. The datalogger was modified by incorporating a radio modem into the existing circuit, installed in a waterproof enclosure at the top of the mounting system's fiberglass pole.

The monitoring and wireless equipment were installed in a soybean field, with a typical soil-moisture monitoring installation shown in Figure 2. The aluminum angle stock was driven into the soil approximately $30 \mathrm{~cm}$ deep, in-line with the soybean plants to minimize exposure to agricultural implements and to avoid tractor and implement wheel-traffic. Installing to a depth of $30 \mathrm{~cm}$ ensured that the remaining $30 \mathrm{~cm}$ of aluminum angle were below the minimum level of any implements likely to be used in the field. An electronics enclosure was bolted to the angle stock to locate it near the ground where it would not be vulnerable to damage, and a cable connecting the radio to the datalogger was secured to the fiberglass pole.
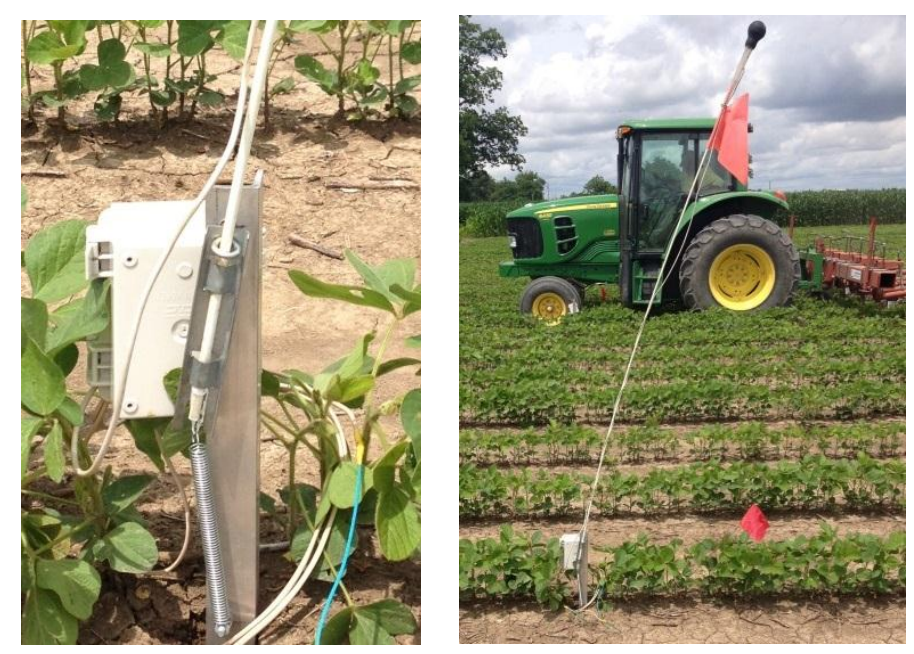

Figure 2. Close-up (Left) and complete view (Right) of field datalogger and radio/antenna mount installed in a soybean field.

Seven wireless soil-moisture monitoring systems were deployed and subjected to encounters with agricultural equipment, including a fertilizer applicator, cultivator, and chemical spray applicator, during 
the 2014 growing season. One such encounter with a tractor pulling a cultivator implement is shown in Figure 3. The fiberglass pole was impacted by the cultivator and flexed to absorb the impact. As the implement traveled over the monitoring site, the fiberglass pole pivoted on the eyebolt and passed safely under the implement. The tension spring was extended, and after the implement had traveled completely beyond the length of the fiberglass pole, contracted to return the radio/antenna mounting system to the vertical position.
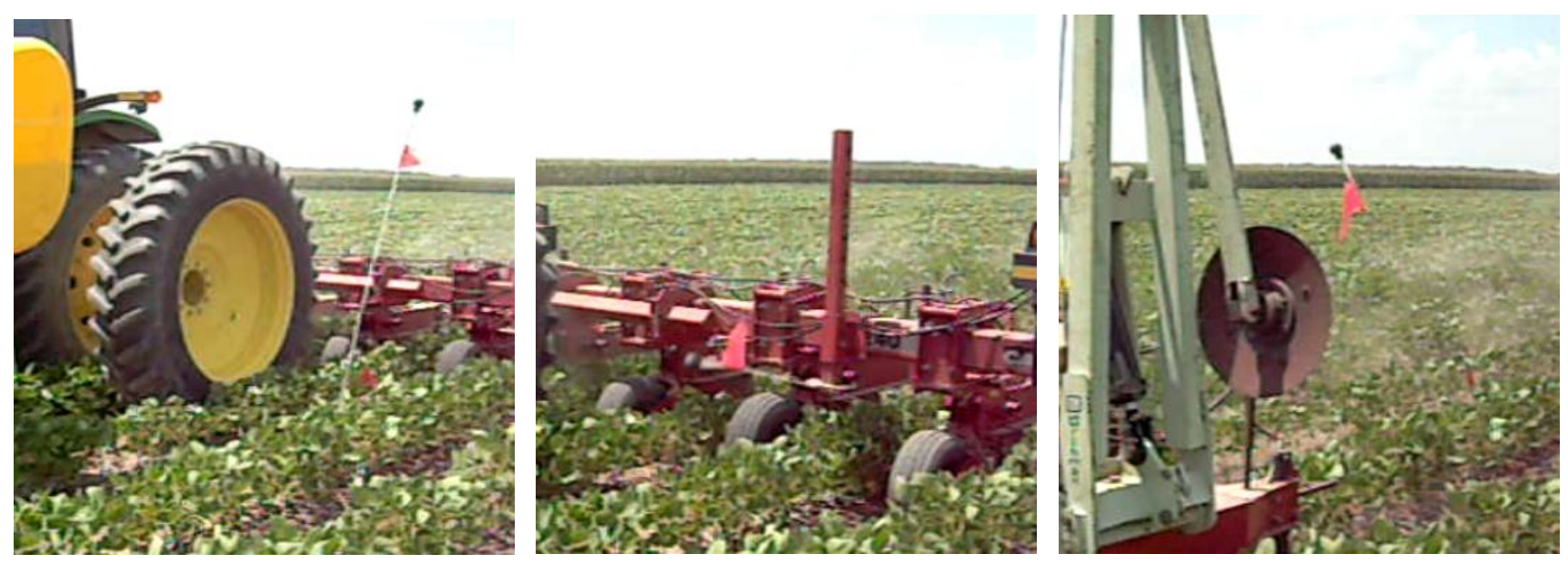

Figure 3. Encounter between radio/antenna mount and cultivator implement before (Left), during (Center), and after (Right) passage of the implement.

\section{Conclusions}

Deployment of wireless monitoring systems is increasing in many diverse environments, including row-crop agricultural fields. To achieve acceptable wireless transmission capability, the radio/antenna must be positioned properly relative to the ground surface or crop canopy to minimize degradation of the radio signal. This requires the mounting of the radio/antenna above the canopy, resulting in potential obstacles to normal agricultural equipment and production operations and damage to the wireless monitoring system. A simple and rugged radio/antenna mounting system was designed which could be subjected to encounters with agricultural equipment and pass under the equipment without suffering physical damage. The mounting system was deployed and tested, and operated successfully following repeated encounters with various agricultural machines and implements. The radio/antenna mount is simple and inexpensive to fabricate using locally available components. While designed to support radio equipment in a wireless network, it could also be used simply as a marker/flag to identify locations in an agricultural field which could be subjected to vehicular traffic or field operations.

\section{Acknowledgments}

This work was supported in part with funding from the Mississippi Soybean Promotion Board.

\section{Conflicts of Interest}

The author declares no conflict of interest. 


\section{References}

1. Wang, N.; Zhang, N.; Wang, M. Wireless sensors in agriculture and food industry-Recent development and future perspective. Comput. Electron. Agric.2006, 50, 1-14.

2. Abbasi, A.Z.; Islam, N.; Shaikh, Z.A. A review of wireless sensors and networks' applications in agriculture. Comput. Stand. Interfaces 2014, 36, 263-270.

3. Fisher, D.K.; Gould, P.J. Open-source hardware is a low-cost alternative for scientific instrumentation and research. Mod. Instrum. 2012, 1, 8-20.

(C) 2015 by the authors; licensee MDPI, Basel, Switzerland. This article is an open access article distributed under the terms and conditions of the Creative Commons Attribution license (http://creativecommons.org/licenses/by/4.0/). 